\title{
Estimation of Displacement and Rotation by Magnetic Tactile Sensor Using Stepwise Regression Analysis
}

\author{
Hiroyuki Nakamoto, Taketo Wakabayashi, Futoshi Kobayashi, and Fumio Kojima \\ Graduate School of System Informatics, Kobe University, Kobe 657-8501, Japan \\ Correspondence should be addressed to Hiroyuki Nakamoto; hiroyuki.nakamoto@kojimalab.com
}

Received 23 June 2014; Accepted 30 September 2014; Published 12 October 2014

Academic Editor: Andrea Cusano

Copyright ( 2014 Hiroyuki Nakamoto et al. This is an open access article distributed under the Creative Commons Attribution License, which permits unrestricted use, distribution, and reproduction in any medium, provided the original work is properly cited.

\begin{abstract}
The human is covered with soft skin and has tactile receptors inside. The skin deforms along a contact surface. The tactile receptors detect the mechanical deformation. The detection of the mechanical deformation is essential for the tactile sensation. We propose a magnetic type tactile sensor which has a soft surface and eight magnetoresistive elements. The soft surface has a permanent magnet inside and the magnetoresistive elements under the soft surface measure the magnetic flux density of the magnet. The tactile sensor estimates the displacement and the rotation on the surface based on the change of the magnetic flux density. Determination of an estimate equation is difficult because the displacement and the rotation are not geometrically decided based on the magnetic flux density. In this paper, a stepwise regression analysis determines the estimate equation. The outputs of the magnetoresistive elements are used as explanatory variables, and the three-axis displacement and the two-axis rotation are response variables in the regression analysis. We confirm the regression analysis is effective for determining the estimate equations through simulation and experiment. The results show the tactile sensor measures both the displacement and the rotation generated on the surface by using the determined equation.
\end{abstract}

\section{Introduction}

The human body is covered with soft skin. We have perceptions of mechanical and thermal stimulation via the skin. In regard to the mechanical stimulation, tactile receptors which are distributed under the skin detect the skin deformation. We recognize the mechanical stimulation based on the tactile receptors' detection and use the recognized stimulation to do various tasks. We cannot conduct any tasks well without tactile sense. The essentiality of the tactile sense indicates necessity of tactile sensors. Therefore, the tactile sensors are expected to have a wide range of application. The tactile sensors provide touch sense to robots, processing machines, intuitive input devices, and texture evaluation and improve intelligence of them.

So as to measure contact states, many tactile sensors have been developed [1]. They have used various principles and combinations of a large variety of components. We can currently buy several tactile sensors $[2,3]$. In particular, most of them have sheet-like structures. Although the sheet-like structure is easy to manufacture, the contact state between the sheet-like sensor and an object has a difference from that between human skin and an object. Because the sheet-like sensor has little deformation by contact, the contact surface is required to be parallel to the sensor surface in order to measure enough. This contact constraints decrease usability of the sheet-like sensors. As described above, the human skin deforms based on contact and recognizes the contact that includes normal and shear deformations. Therefore, the softness and deformation of tactile sensors are essential for multiaxis tactile sensors.

Various tactile sensors which have soft surface and multiaxis sensitivity have been proposed: sensors using a strain gauge or a PVDF film, capacitive sensors, optical sensors, magnetic sensors, and sensors using a tomography technique [4]. With regard to optical tactile sensors, the methods which capture images of contact surfaces and calculate the deformation of the surface have been proposed. Ferrier and 
Brockett captured inside of thin film by a pinhole camera and calculated the deformation of the thin film from the pattern of dots on the film [5]. Yussof et al. proposed a sensor of a hemisphere face with many elastomer contacts [6]. The pattern of the contacts determined three-axis force. Although these optical tactile sensors have an advantage which has no wiring inside the soft surface, downsizing of camera devices and high-speed calculation are required. In addition, Tactile sensors based on change in capacitance have been reported. Hoshi and Shinoda proposed a tactile sensor that is composed of two urethane forms and three pieces of conductive fabric [7]. Lipomi et al. proposed a flexible sensor that was made of an elastomer and carbon nanotubes [8]. This sensor measured relative change of capacitance in the compression of it. Although the elastomer includes electrodes and is flexible, the change of capacitance is too small to reduce external noise. As referred to above, soft surface and multiaxis tactile sensors based on various measurement principles have been proposed. Their improvement is expected for practical use.

In regard to magnetic tactile sensors, Nowlin proposed a tactile sensor with hall elements [9]. To reduce noise, this sensor used a stochastic method based on elaborate calculation. A tactile sensor using induction coils was proposed by Takenawa [10]. The output voltage of the coils was small and has drift instability. The magnetic tactile sensors need much calculation because the magnetic elements have nonlinear characteristics. At the same time, an absence of cable between magnet and magnetic elements is a major advantage to realize simple structure of sensor.

In this study, we have proposed a tactile sensor using a permanent magnet and giant magnetoresistive (GMR) elements [11, 12]. The GMR elements convert change in magnetic flux density to output voltage. The tactile sensor measured three-axis displacement applied on the surface. However, the contact surface was limited to being parallel to the tactile sensor in order to use a simple calculation. In the case of being not parallel, the output of the tactile sensor has errors. In this paper, we propose a magnetic type tactile sensor that measures three-axis displacement and two-axis rotation applied on the surface of it. After describing the structure of the tactile sensor and its problem, we propose a determining method of a regression equation. Explanatory variables in the equation are the output voltages of the GMR elements, and response variables are values of the displacement and the rotation. To confirm the effectiveness of the proposed method, we verify results of both simulation and experiment.

\section{Magnetic Type Tactile Sensor}

2.1. Structure. The structure of the tactile sensor is shown in Figure 1. The sensor is mainly composed of two layers, that is, an elastic layer and a substrate layer. The elastic layer is made of an elastic material, for example, urethane elastomer, and includes a cylindrical permanent magnet inside. The substrate layer is made of a glass epoxy board; its surface side is flat and contains no electronic elements. The GMR

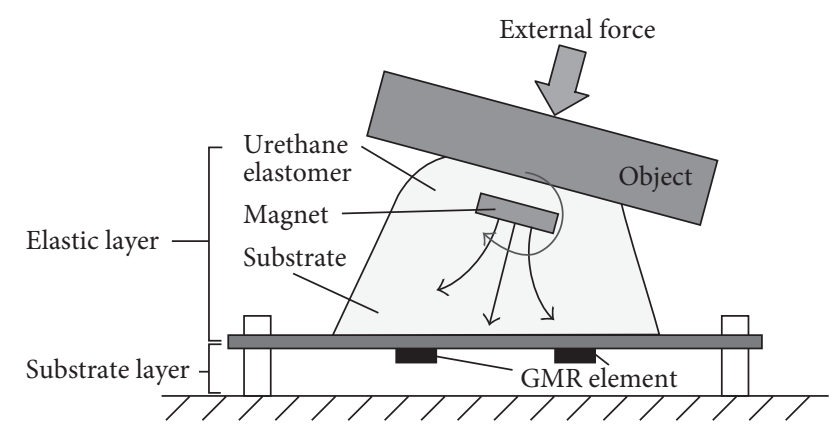

FIGURE 1: Sensor structure.

elements are on the back side. The elastic layer is fixed to the substrate layer's surface with an adhesive bond. Because these layers are not hard-wired, no wire breakages occur. If the elastic layer is worn away after a long-term usage, it can be easily replaced with a new one.

2.2. Measurement Principle. When the tactile sensor touches an object as shown in Figure 1, the contact deforms the surface of the elastic layer. The magnet inside the elastic layer is also displaced depending on the degree to which the layer is deformed. This magnet displacement changes the magnetic flux density to the GMR elements on the substrate layer, and thus the outputs of the elements are changed. Based on the outputs, the sensor estimates three-axis displacement and two-axis rotation of the layer surface.

To determine the surface displacement from the outputs of the GMR elements, we proposed an estimate equation of $x$-axis displacement expressed in (1) [12]. $v_{i}(i=1 \cdots 4)$ indicates the outputs of the four GMR elements:

$$
\begin{aligned}
\Delta x= & C_{d x 1}+\frac{C_{d x 2}}{v_{1}^{2}}+\frac{C_{d x 3}}{v_{1}}+\frac{C_{d x 4}}{v_{2}^{2}}+\frac{C_{d x 5}}{v_{2}} \\
& +\frac{C_{d x 6}}{v_{3}^{2}}+\frac{C_{d x 7}}{v_{3}}+\frac{C_{d x 8}}{v_{4}^{2}}+\frac{C_{d x 9}}{v_{4}}
\end{aligned}
$$

where $C_{d x j}(j=1 \cdots 9)$ is a coefficient of each term, and it is determined by a multiple regression analysis. The equation is effective in the case that the magnet is displaced parallel to the substrate. Therefore, when the magnet rotates in the elastic layer as shown in Figure 1, $\Delta x$ in (1) includes an error caused by the rotation. To estimate the contact surface correctly, a novel regression equation that estimates both three-axis displacement and two-axis rotation is required. In this study, we determine the regression equation by a stepwise regression analysis.

\section{Method for Determining Regression Equation}

3.1. Stepwise Regression Analysis. To estimate both displacement and rotation based on the outputs of the GMR elements in the tactile sensor, a stepwise method determines a regression equation. In this method, response variables 
are five, that is, the three-axis displacement and the twoaxis rotation which occurred on the surface of the sensor. Candidates of explanatory variable are the variables based on the output voltages of the GMR elements. The stepwise regression analysis selects the explanatory variables from the candidates to estimate the response variables. This selection is performed based on Akaike's information criterion (AIC). The AIC is expressed by

$$
\mathrm{AIC}=n \log _{e} S_{e}+2 p,
$$

where $p$ is the number of the explanatory variables used in the regression equation, $n$ is the number of data set of measurement, and $S_{e}$ indicates the residual sum of squares between the measurement data and the output of the regression equation. When the AIC is a small value, the balance of the number of the explanatory variables and the accuracy of the estimation is in a good condition. Now, the accuracy is determined based on the residual sum of squares between the measurement data and the output of the regression equation and is expressed with the first term in (2). Assuming the regression equation uses many explanatory variables, the accuracy of the estimation would be high. In that case, the equation has a possibility of including unnecessary explanatory variables. To estimate both the three-axis displacement and the two-axis rotation in a short time, the less number of explanatory variables is suitable. Therefore, the regression equation is determined by minimizing the AIC.

3.2. Determination of Regression Equation. In advance, the data set, which includes the outputs of the GMR elements, the displacements $(\Delta x, \Delta y, \Delta z)$, and the rotations $\left(\theta_{x}, \theta_{y}\right)$, is obtained in order to determine the regression equation. The tactile sensor has eight GMR elements. Their output voltages are expressed as $v_{k}(k=1 \cdots 8)$. We defined the candidates of the explanatory variables as follows:

$$
v_{k}, v_{k}^{2}, v_{k}^{3}, v_{k}^{4}, \frac{1}{v_{k}}, \frac{1}{v_{k}^{2}}, \frac{1}{v_{k}^{3}}, \frac{1}{v_{k}^{4}}, v_{k}^{1 / 2}, v_{k}^{1 / 3}, v_{k}^{1 / 4} .
$$

The stepwise regression selects the explanatory variables from these candidates. The selected explanatory variables are evaluated by the AIC. The algorithm for determining the regression equation is indicated as the following steps.

(1) Use (1) as the initial regression equation, and define its explanatory variables as the initial variables.

(2) Define the variables in the regression equation as the explanatory variables and the others as the candidates.

(3) Calculate the AIC by using the regression equation in the case that one of the candidates is added to the explanatory variables. The calculations are carried out to all the candidates.

(4) Calculate the AIC by using the regression equation in the case that one of the explanatory variables is removed from the equation. The calculations are carried out to all the explanatory variables.

(5) In regard to the results of (3) and (4), after determining the regression equation that has the explanatory variables of the minimal AIC, return to (2). If the minimal AIC is higher than that of the previous trial, proceed to (6).

(6) As the regression equation, determine the equation composed of the explanatory variables defined in (2).

The response variables are $\Delta x, \Delta y, \Delta z, \Delta \theta_{x}$, and $\Delta \theta_{y}$. Because $\Delta \theta_{x}$ and $\Delta \theta_{y}$ are much affected by $\Delta x, \Delta y$, and $\Delta z$, the candidates of the explanatory variables $\Delta \theta_{x}$ and $\Delta \theta_{y}$ include the following variables using $\Delta x, \Delta y$, and $\Delta z$ :

$$
\Delta x, \Delta y, \Delta z, v_{k} \Delta x, v_{k} \Delta y, v_{k} \Delta z \text {. }
$$

The number of the candidates' variables is 17 kinds in the case of without counting $k$ of $v_{k}$. Because the regression equations of $\Delta \theta_{x}$ and $\Delta \theta_{y}$ need the estimates of $\Delta x, \Delta y$, and $\Delta z$, first, this determination procedure determines the explanatory variables of the displacement $(\Delta x, \Delta y$, and $\Delta z)$. Second, those of the rotation $\left(\Delta \theta_{x}\right.$ and $\left.\Delta \theta_{y}\right)$ are determined.

\section{Simulation and Experiment}

4.1. Sensor Design. We designed the substrate of the tactile sensor. To estimate the three-axis displacement and the twoaxis rotation on the contact surface, the substrate has the eight GMR elements upon the bottom side. The drawings are shown in Figure 2. The GMR elements (AA003-02, NVE Co.) are arranged on a circular line of $10 \mathrm{~mm}$ radius not to have aeolotropy. They have a one-direction sensitivity. Their directions are the radial directions of the circle. The substrate is a glass epoxy board. Its thickness is $1.5 \mathrm{~mm}$. The position of the inside magnet and the size of the elastic layer are shown in Figure 3. The magnet is a cylindrical neodymium magnet. Its size is $6 \mathrm{~mm}$ in diameter and $1 \mathrm{~mm}$ in thickness. The space between the bottom of the magnet and the top of the substrate is $11 \mathrm{~mm}$. The elastic layer is a circular truncated cone and is made from urethane. The bottom is $20 \mathrm{~mm}$ in diameter, and the top side is $16 \mathrm{~mm}$ in diameter. The parameters of the design were determined by a finite element method [11].

4.2. Simulation. To confirm the effectiveness of the stepwise regression analysis, we determined a regression equation based on simulation data and evaluated the estimation accuracy of the displacement and rotation of the magnet. Based on the design of the tactile sensor, we calculated the magnetic flux density from the cylindrical magnet at the positions of the GMR elements. In the calculation, the magnet changed its displacement and its rotation as follows:

(i) $\Delta x, \Delta y:-2,-1,0,1,2 \mathrm{~mm}$;

(ii) $\Delta z: 0,-1,-2 \mathrm{~mm}$;

(iii) $\Delta \theta_{x}, \Delta \theta_{y}:-5,-2.5,0,2.5,5 \mathrm{deg}$

The origin is the center of the top side of the elastic layer as indicated in Figure 3. Each data set is composed of eight magnetic flux densities, $\Delta x, \Delta y, \Delta z, \Delta \theta_{x}$, and $\Delta \theta_{y}$. Because the number of the combinations in the data set is the multiplication of the numbers of $\Delta x, \Delta y, \Delta z, \Delta \theta_{x}$, and $\Delta \theta_{y}$, the number of the data set is 1875 . The stepwise regression 


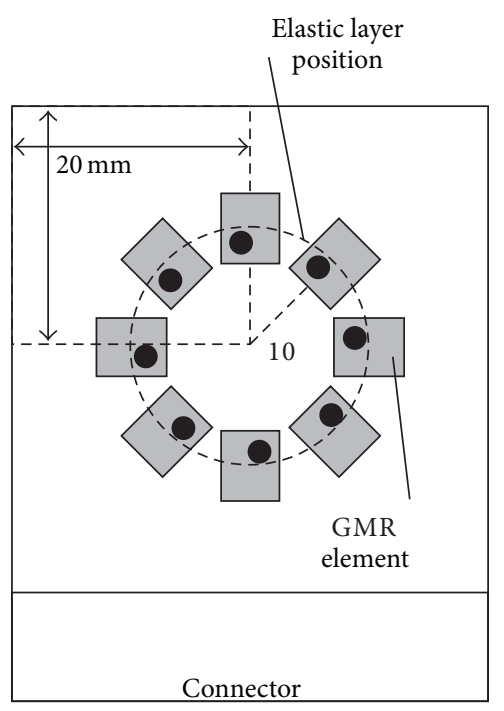

Figure 2: Design of substrate.

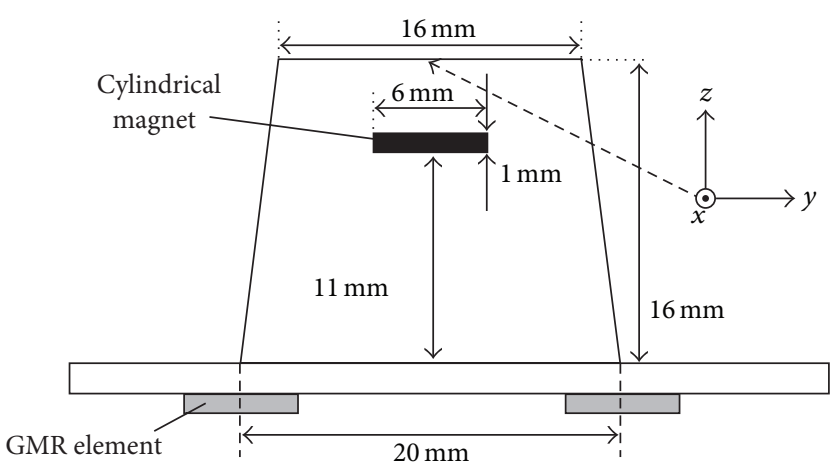

FIGURE 3: Cross-section view of sensor.

analysis determined the regression equations of $\Delta x, \Delta y, \Delta z$, $\Delta \theta_{x}$, and $\Delta \theta_{y}$ based on the data set.

$\Delta x$ and $\Delta y$ have a symmetrical relationship. $\Delta \theta_{x}$ and $\Delta \theta_{y}$ likewise have a rotational symmetry. Because they have the same results, the results of $\Delta x, \Delta z$, and $\Delta \theta_{x}$ are shown in Figures 4, 5, and 6, respectively. Each horizontal axis indicates an index of the data set. The indexes between the different charts have no relationship. In the charts, the estimate value and the target value which have the same index correspond to each other. The estimate values of $\Delta x$ had a good agreement with the target values in Figure 4. The maximal error of $\Delta x$ was $0.06 \mathrm{~mm}$. The estimate values of $\Delta z$ coincided approximately with the target values at $\Delta z=0$ and -1 . At $\Delta z=-2$, the errors were larger than those at $\Delta z=0$ and -1 . The maximal error of $\Delta z$ was $0.25 \mathrm{~mm}$. The results indicate the determined regression equation reduced the influence of the rotation of the magnet for the threeaxis displacement. The estimate results of $\Delta \theta_{x}$ had the same trend with those of $\Delta z$ and had the large errors at $\Delta \theta_{x}= \pm 5$. The maximal error of $\Delta \theta_{x}$ was $1.23 \mathrm{deg}$. The resolution of $\Delta \theta_{x}$ was also evaluated and is shown in Figure 6. Figure 7

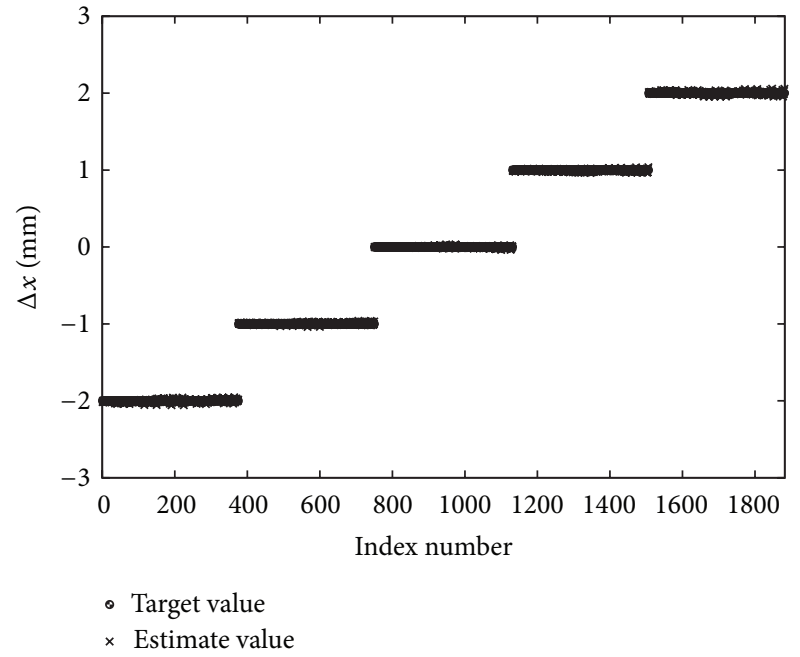

FIGURE 4: Simulation result: displacement in $x$-axis direction.

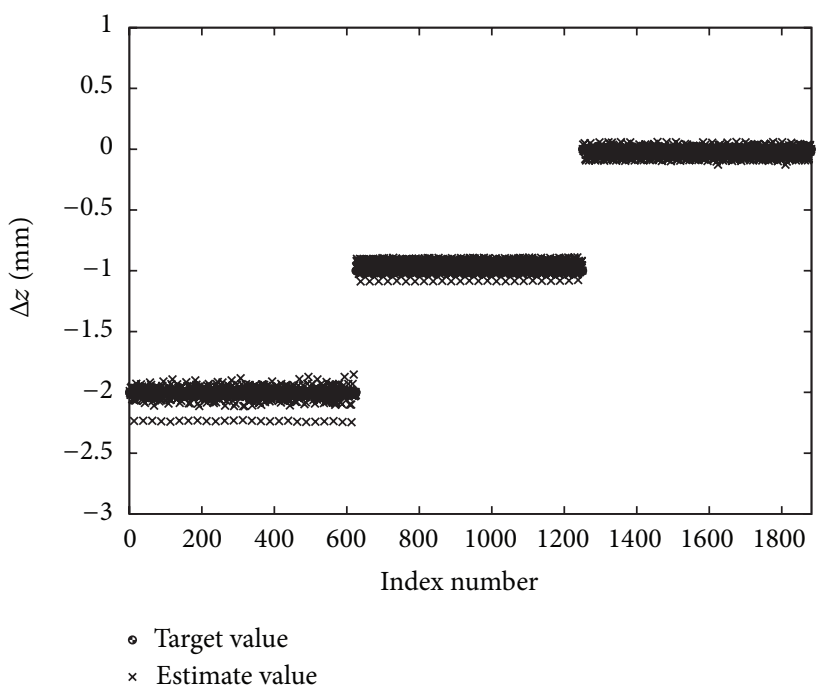

FIGURE 5: Simulation result: displacement in $z$-axis direction.

shows the estimated rotation $\Delta \theta_{x}$ of $0.1 \mathrm{deg}$ per one step. The other response variables were set at 0 . Although the error at $5 \mathrm{deg}$ was $1 \mathrm{deg}$, the slope is approximately constant. This result indicates the relative resolution is high. Through the simulation, we confirmed that the stepwise regression analysis determined the regression equation estimating the displacement and the rotation of the magnet.

4.3. Experiment. In the simulation, although the displacement and rotation of the magnet are calculated without the elastic layer model, the stepwise regression analysis based on the AIC determined the regression equation that estimates the displacement and the rotation of the magnet. Assuming the magnet has the displacement and the rotation depending on the top surface of the elastic layer, the stepwise regression analysis is also effective to an actual tactile sensor. Thus, we fabricated a tactile sensor and performed experiments. 


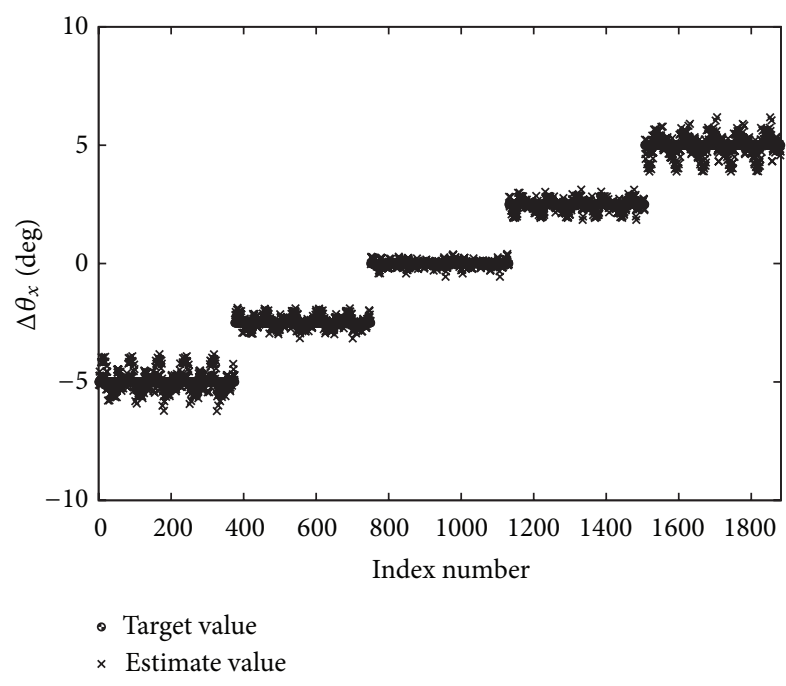

FIGURE 6: Simulation result: rotation around $x$-axis.

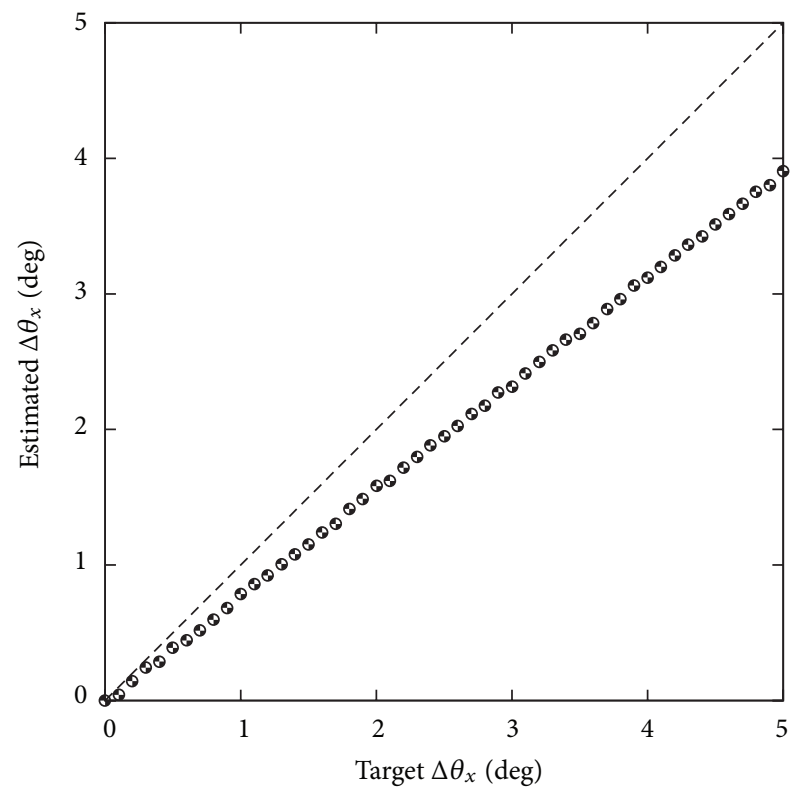

FIGURE 7: Simulation result: rotation around $x$-axis of 0.1 deg per one step.

The tactile sensor is shown in Figure 8. The size or the other parameters of the sensor were along those values in Figures 2 and 3 . To generate the displacement and the rotation with high accuracy, we used a motorized stage (SGSP26-100, Sigma Koki Co.) in the experiments. As shown in Figure 9, The stage has three translation axes and two rotation axes and touches the tactile sensor with a flat plate. The flat plate displaced and tilted the top surface of the tactile sensor. The ranges of the displacement and rotation are as follows:

(i) $\Delta x, \Delta y:-2,-1,0,1,2 \mathrm{~mm}$;

(ii) $\Delta z:-2,-3 \mathrm{~mm}$;

(iii) $\Delta \theta_{x}, \Delta \theta_{y}:-5,0,5 \mathrm{deg}$.

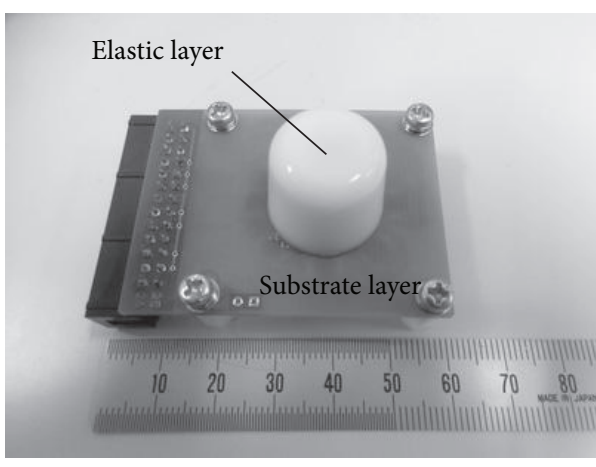

Figure 8: Fabricated tactile sensor.

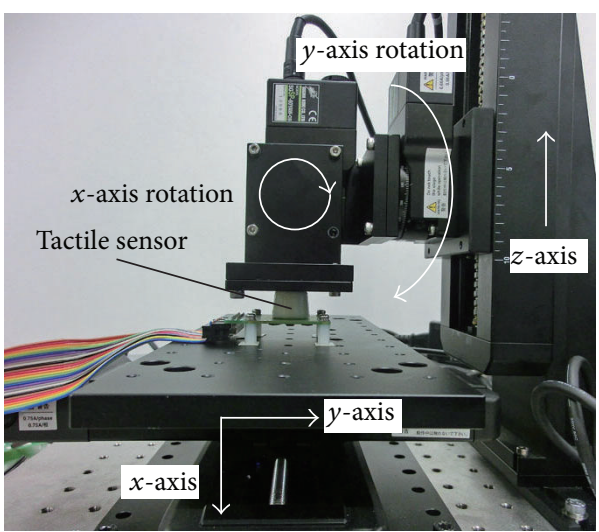

Figure 9: Experimental setup.

The number of the combination of the displacement and the rotation was 450 . Since the displacement of $\Delta z=-1$ was too small to rotate the contact surface, we excepted it from the combination. The output voltages of the eight GMR elements were measured at each combination. The data set composed of the GMRs' outputs, $\Delta x, \Delta y, \Delta z, \Delta \theta_{x}$, and $\Delta \theta_{y}$, was also 450 . The stepwise regression analysis determined the regression equations based on the data set. Next, the experimental setup measured another data set. Using the additional data set, we verified $\Delta x, \Delta y, \Delta z, \Delta \theta_{x}$, and $\Delta \theta_{y}$ estimated by the determined regression equations.

Because the results of $\Delta y$ and $\Delta \theta_{y}$ had the same trends with $\Delta x$ and $\Delta \theta_{x}$, respectively, Figures 10,11 , and 12 show the results of $\Delta x, \Delta z$, and $\Delta \theta_{x}$, respectively. In contrast to the simulation result in Figure 4, Figure 10 shows that $\Delta x$ had errors. The maximal error was $0.75 \mathrm{~mm}$. Although these results were improved from the results by (1), the maximal error was so high that an application of the tactile sensor to a precision measurement is difficult. One of the causes was a decay of the displacement of the magnet by the elastic layer. In that case, the changes of the explanatory variables were not enough for the estimation of the displacement. On the other hand, $\Delta z$ and $\Delta \theta_{x}$ had small errors. The maximal errors of $\Delta z$ and $\Delta \theta_{x}$ were $0.13 \mathrm{~mm}$ and $0.95 \mathrm{deg}$. The stepwise regression analysis was effective in regards to $\Delta z$ and $\Delta \theta_{x}$. The numbers of the explanatory variables of $\Delta x, \Delta z$, and $\Delta \theta_{x}$ were 18,21 , and 39, respectively. The regression equations of $\Delta x, \Delta z$, and 


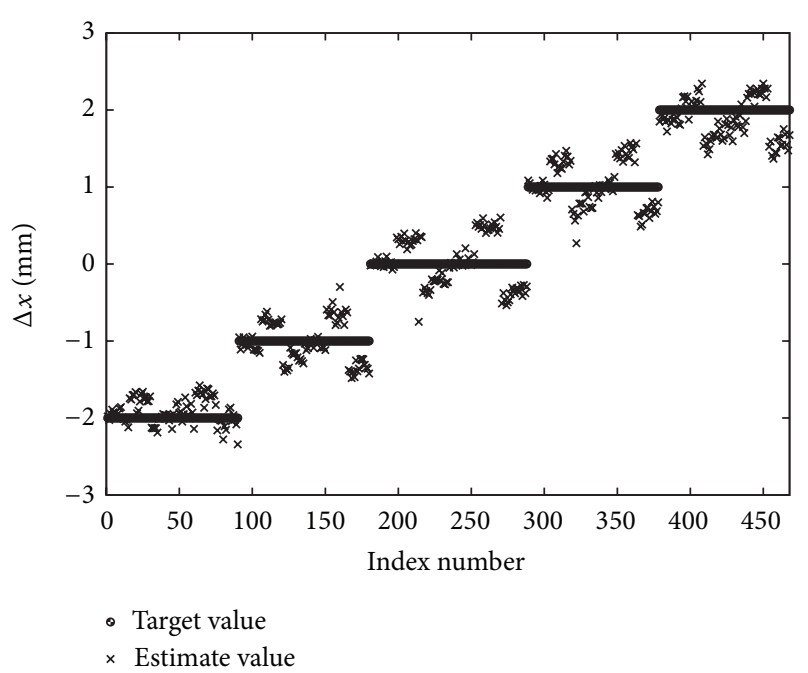

FIGURE 10: Experimental result: displacement in $x$-axis direction.

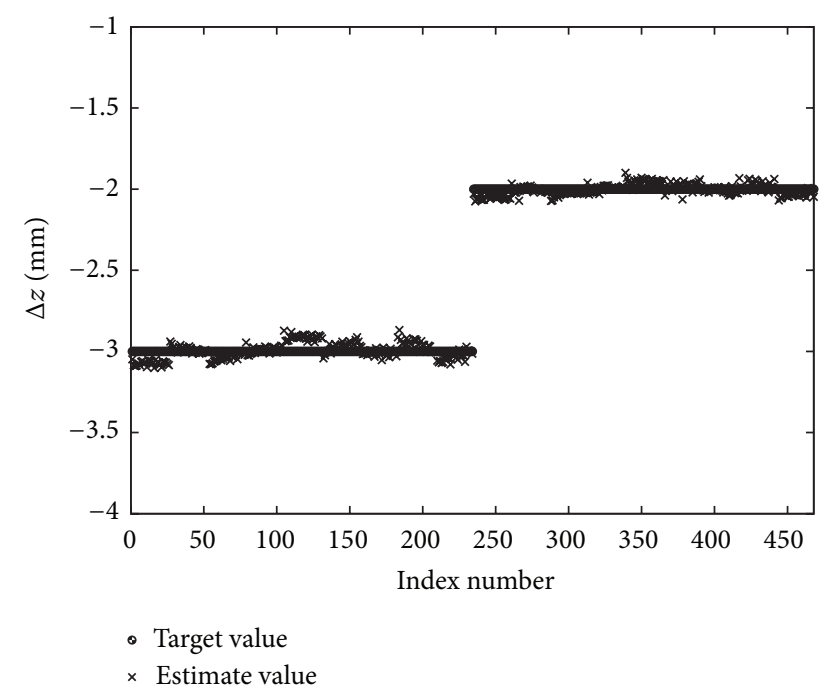

FIGURE 11: Experimental result: displacement in $z$-axis direction.

$\Delta \theta_{x}$ are shown in the Appendix. Because the numbers of the explanatory variables indicate complexity of estimation, the results show the estimation of $\Delta \theta_{x}$ was difficult.

The resolution of $\Delta \theta_{x}$ is shown in Figure 13. Although the estimated $\Delta \theta_{x}$ had the small variation, the trend was coincident with the result of the simulation. The estimated $\Delta \theta_{x}$ has the large difference with the target $\Delta \theta_{x}$ at the high value. One of the causes is that the variation of the explanatory variables is not enough. An addition of other explanatory variables is necessary to improve the results.

\section{Conclusions}

In order to realize a flexible and multiaxis tactile sensor, the stepwise regression analysis determined the regression equations that estimate the three-axis displacement and the two-axis rotation on the tactile sensor with the eight GMR

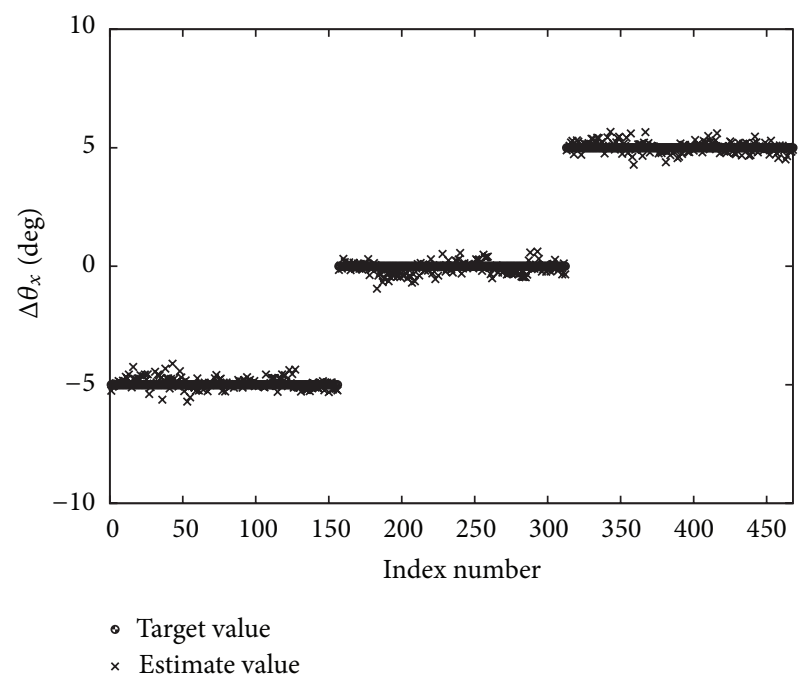

FIGURE 12: Experimental result: rotation around $x$-axis.

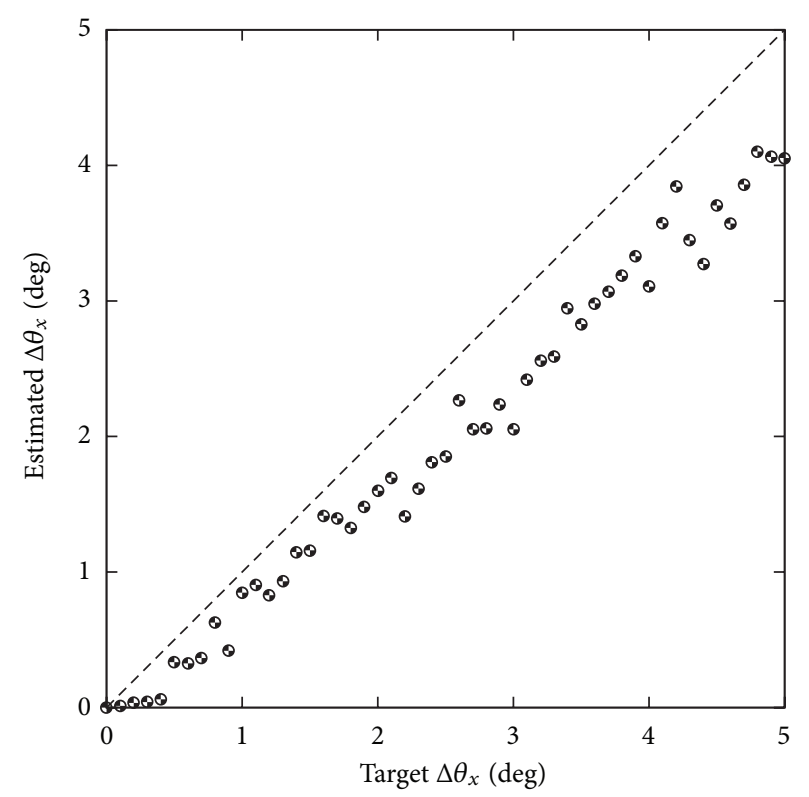

FIGURE 13: Experimental result: rotation around $x$-axis of 0.1 deg per one step.

elements. The response variables were the values of the displacement and the rotation of the sensor surface, and the candidates of the explanatory variables were composed of the outputs of the GMR elements. The stepwise regression analysis evaluated the explanatory variables by the AIC. First, the effectiveness of the method was evaluated in the simulation. The maximal error was $0.25 \mathrm{~mm}$ in displacement and $1.23 \mathrm{deg}$ in rotation. Second, we conducted the experiments using the tactile sensor. In these results, we confirmed that the maximal error was $0.75 \mathrm{~mm}$ in displacement and $0.95 \mathrm{deg}$ in rotation. The stepwise regression analysis determined the regression equations that estimate the displacement and the rotation of the surface of the tactile sensor. 
The experimental results were not enough to apply the tactile sensor for a precision measurement. So as to improve the accuracy of the displacement and the rotation, there are two ways. The GMR elements were placed on the same plane in this study, a three-dimensional configuration of them has a possibility to improve the accuracy. In addition, although the proposed method used the 17 candidates of the explanatory variables, they are not enough for expressing the response variables with a high accuracy. We need select the candidates of the explanatory variables based on the physical relation between the magnet and the GMR elements. They are a future work of our study.

\section{Appendix}

The regression equations decided on the experiment are shown as follows. Because the equations of $\Delta y$ and $\Delta \theta_{y}$ had the same trends with $\Delta x$ and $\Delta \theta_{x}$, respectively, only the equations of $\Delta x, \Delta z$, and $\Delta \theta_{x}$ are shown:

$$
\begin{aligned}
& \Delta x=-6.1+\frac{453.9}{v_{1}}-\frac{981.1}{v_{3}}-\frac{195.4}{v_{4}}+\frac{132.5}{v_{6}}+\frac{66.0}{v_{7}} \\
& +\frac{49.9}{v_{8}}-\frac{1006.0}{v_{1}^{2}}-\frac{62.5}{v_{2}^{2}}+\frac{2103.1}{v_{3}^{2}}+\frac{189.1}{v_{4}^{2}} \\
& +\frac{33.5}{v_{5}^{2}}-\frac{145.0}{v_{6}^{2}}-\frac{968.5}{v_{8}^{2}}-\frac{2750.0}{v_{3}^{4}}+\frac{1477.2}{v_{1}^{4}} \\
& +\frac{975.2}{v_{8}^{4}}+0.024 v_{2}^{4} \\
& \Delta z=-32.2+\frac{225.8}{v_{1}}+\frac{8.3}{v_{2}}-\frac{105.7}{v_{3}}+\frac{60.9}{v_{4}}-\frac{45.7}{v_{5}} \\
& +\frac{123.1}{v_{6}}-\frac{75.0}{v_{7}}-\frac{102.5}{v_{8}}-\frac{422.9}{v_{1}^{2}}+\frac{233.0}{v_{3}^{2}} \\
& -\frac{93.1}{v_{4}^{2}}+\frac{77.6}{v_{5}^{2}}-\frac{152.0}{v_{6}^{2}}+\frac{190.2}{v_{7}^{2}}+\frac{153.3}{v_{8}^{2}} \\
& +0.41 v_{6}^{2}-0.006 v_{8}^{4}+\frac{407.3}{v_{1}^{4}}-\frac{319.0}{v_{3}^{4}}-\frac{246.2}{v_{7}^{4}}, \\
& \Delta \theta_{x}=-13073.9-\frac{479.3}{v_{2}}+\frac{292.1}{v_{3}}-\frac{888.8}{v_{4}} \\
& -\frac{4121.1}{v_{5}}+\frac{969175.1}{v_{6}}+\frac{587.4}{v_{7}}-\frac{15047.9}{v_{8}} \\
& -\frac{630.5}{v_{1}^{2}}+\frac{894.2}{v_{4}^{2}}-\frac{6802.0}{v_{5}^{2}}-\frac{2835023}{v_{6}^{2}}+\frac{1546.1}{v_{8}^{2}} \\
& -36.1 \Delta y+4.9 v_{8} \Delta y+3.4 v_{6} \Delta y-4.3 v_{8} \Delta x \\
& +3.7 v_{7} \Delta x-0.26 v_{1}^{4}+487.4 v_{7}^{1 / 4}-74.1 v_{6}^{4}+5.79 v_{4}^{4} \\
& +9.69 v_{8}^{4}-58.6 v_{8}^{3}-\frac{2130609}{v_{6}^{4}}-13.9 \Delta x \\
& -106.1 \Delta z+17.4 v_{6} \Delta z+59.6 v_{6}^{3}+3.1 v_{7} \Delta y \\
& +9.1 v_{4} \Delta z+\frac{3926535}{v_{6}^{3}}-8.3 v_{3} \Delta z-3.0 v_{3} \Delta y \\
& -34.7 v_{4}^{3}+19.2 v_{5} \Delta z+4.9 v_{1} \Delta x-\frac{5840.4}{v_{5}^{4}}+\frac{1158.0}{v_{1}^{4}} \text {. }
\end{aligned}
$$

\section{Conflict of Interests}

The authors declare that there is no conflict of interests regarding the publication of this paper.

\section{Acknowledgment}

This work was supported by a research grant of The JGC-S Scholarship Foundation.

\section{References}

[1] M. H. Lee and H. R. Nicholls, "Tactile sensing for mechatronics-a state of the art survey," Mechatronics, vol. 9, no. 1, pp. 1-31, 1999.

[2] Tekscan web site, http://www.tekscan.com/.

[3] Pressure Profile Systems web site, http://www.pressureprofile .com/.

[4] M. R. Cutkosky, R. D. Howe, and W. R. Provancher, "Force and tactile sensors," in Springer Handbook of Robotics, pp. 455-476, Springer, Berlin, Germany, 2008.

[5] N. J. Ferrier and R. W. Brockett, "Reconstructing the shape of a deformable membrane from image data," The International Journal of Robotics Research, vol. 19, no. 9, pp. 795-816, 2000.

[6] H. Yussof, J. Wada, and M. Ohka, "Object handling tasks based on active tactile and slippage sensations in a multi-fingered humanoid robot arm," in Proceedings of the IEEE International Conference on Robotics and Automation (ICRA '09), pp. 502507, May 2009.

[7] T. Hoshi and H. Shinoda, "Robot skin based on touch-areasensitive tactile element," in Proceedings of the IEEE International Conference on Robotics and Automation (ICRA '06), pp. 3463-3468, May 2006.

[8] D. J. Lipomi, M. Vosgueritchian, B. C.-K. Tee et al., "Skin-like pressure and strain sensors based on transparent elastic films of carbon nanotubes," Nature Nanotechnology, vol. 6, no. 12, pp. 788-792, 2011.

[9] W. C. Nowlin, "Experimental results on Bayesian algorithms for interpreting compliant tactile sensing data," in Proceedings of the IEEE International Conference on Robotics and Automation, vol. 1, pp. 378-383, Sacramento, Calif, USA, April 1991.

[10] S. Takenawa, "A magnetic type tactile sensor using a twodimensional array of inductors," in Proceedings of the IEEE International Conference on Robotics and Automation (ICRA '09), pp. 3295-3300, Kobe, Japan, May 2009.

[11] H. Nakamoto, M. Goka, S. Takenawa, and Y. Kida, "Development of tactile sensor using magnetic elements," in Proceedings of the IEEE Workshop on Robotic Intelligence in Informationally Structured Space (RIISS '11), pp. 37-42, Paris, France, April 2011.

[12] M. Goka, H. Nakamoto, and S. Takenawa, "A magnetic type tactile sensor by GMR elements and inductors," in Proceedings of the 23rd IEEE/RSJ 2010 International Conference on Intelligent Robots and Systems (IROS '10), pp. 885-890, October 2010. 

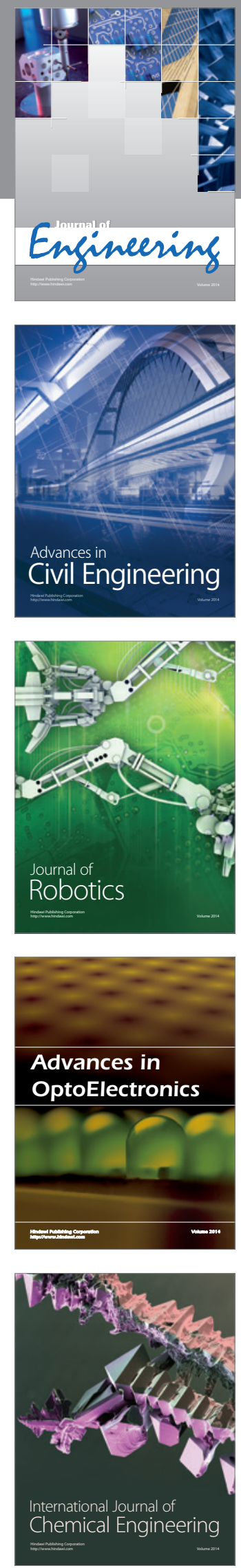

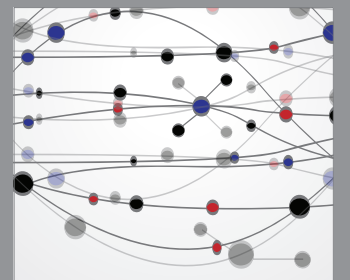

The Scientific World Journal
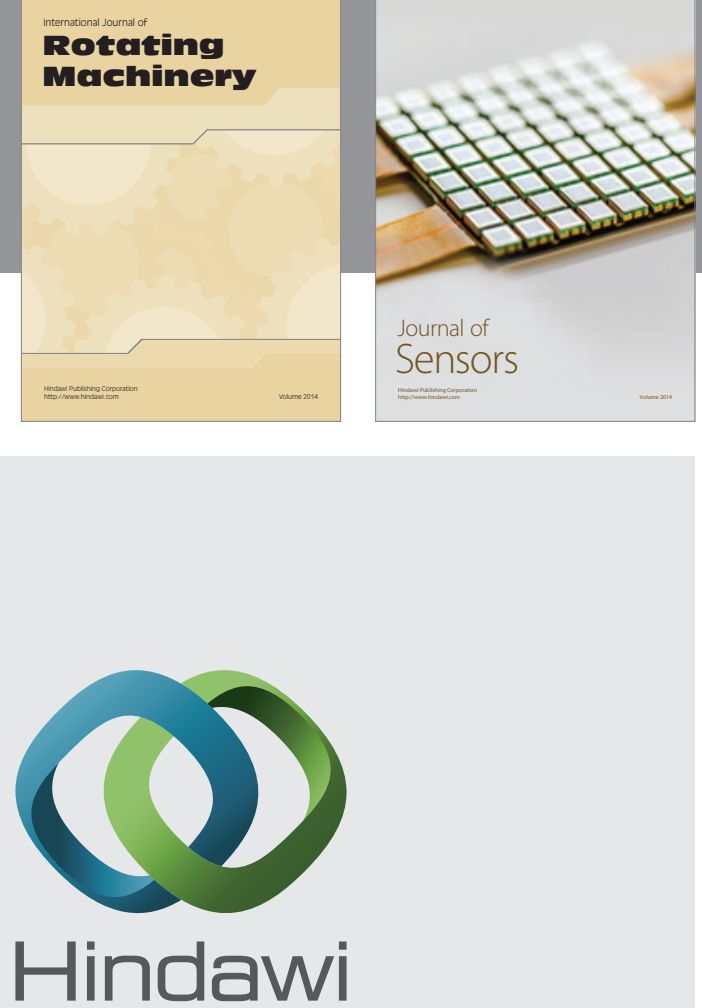

Submit your manuscripts at http://www.hindawi.com
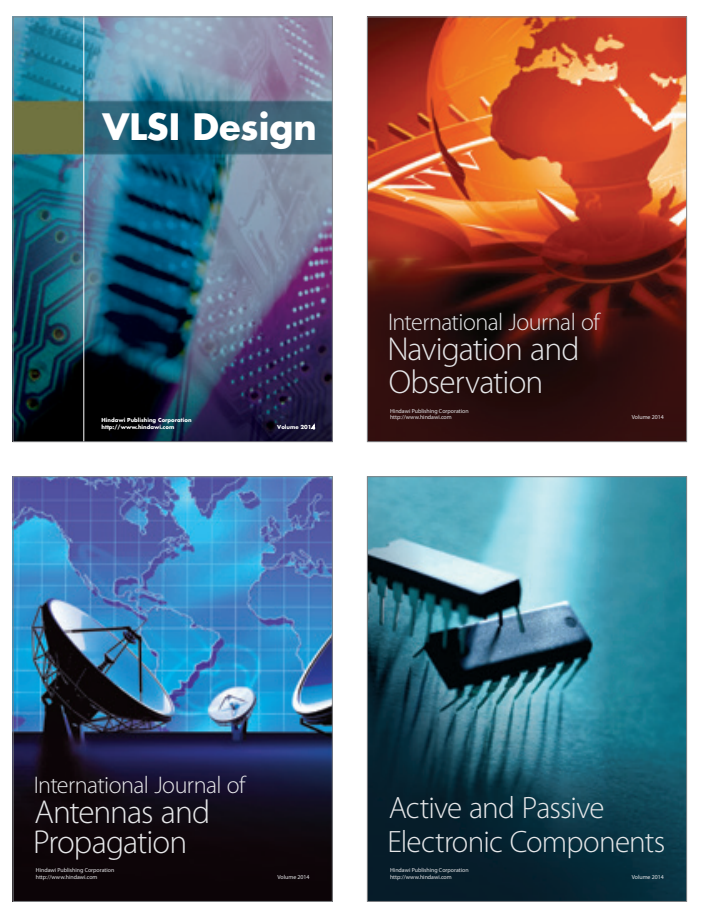
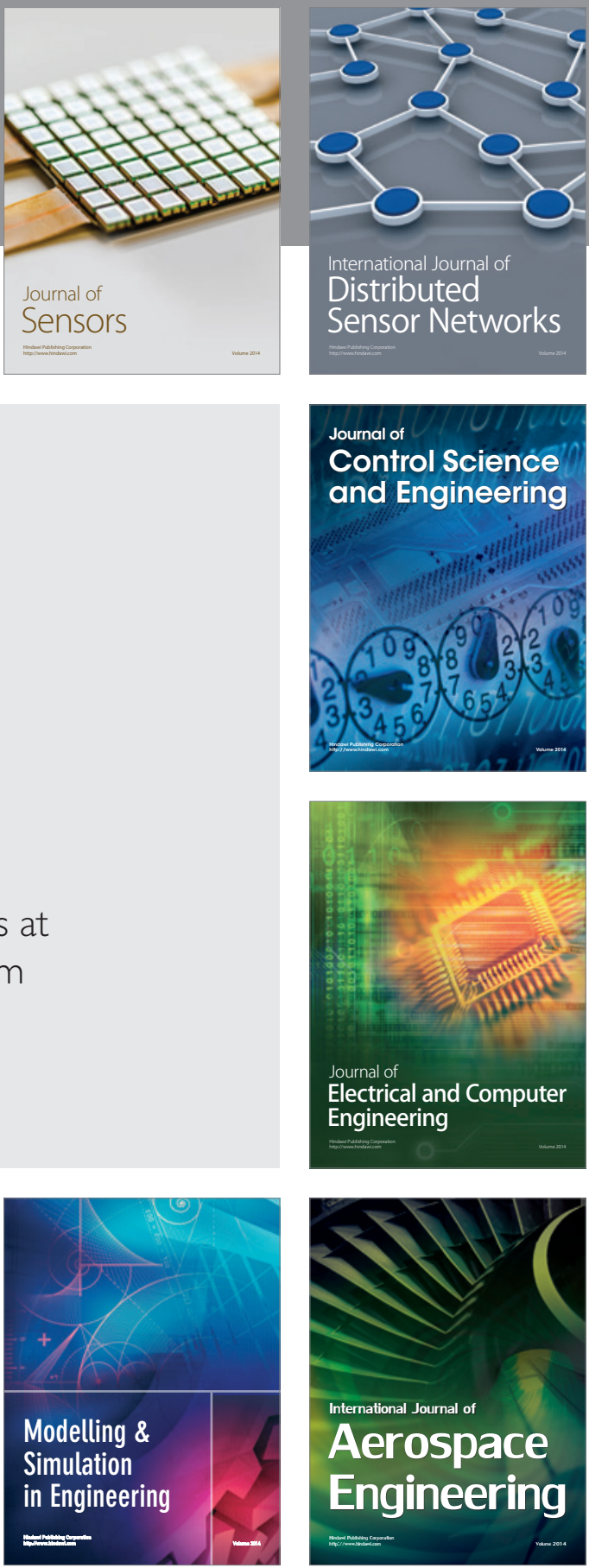

Journal of

Control Science

and Engineering
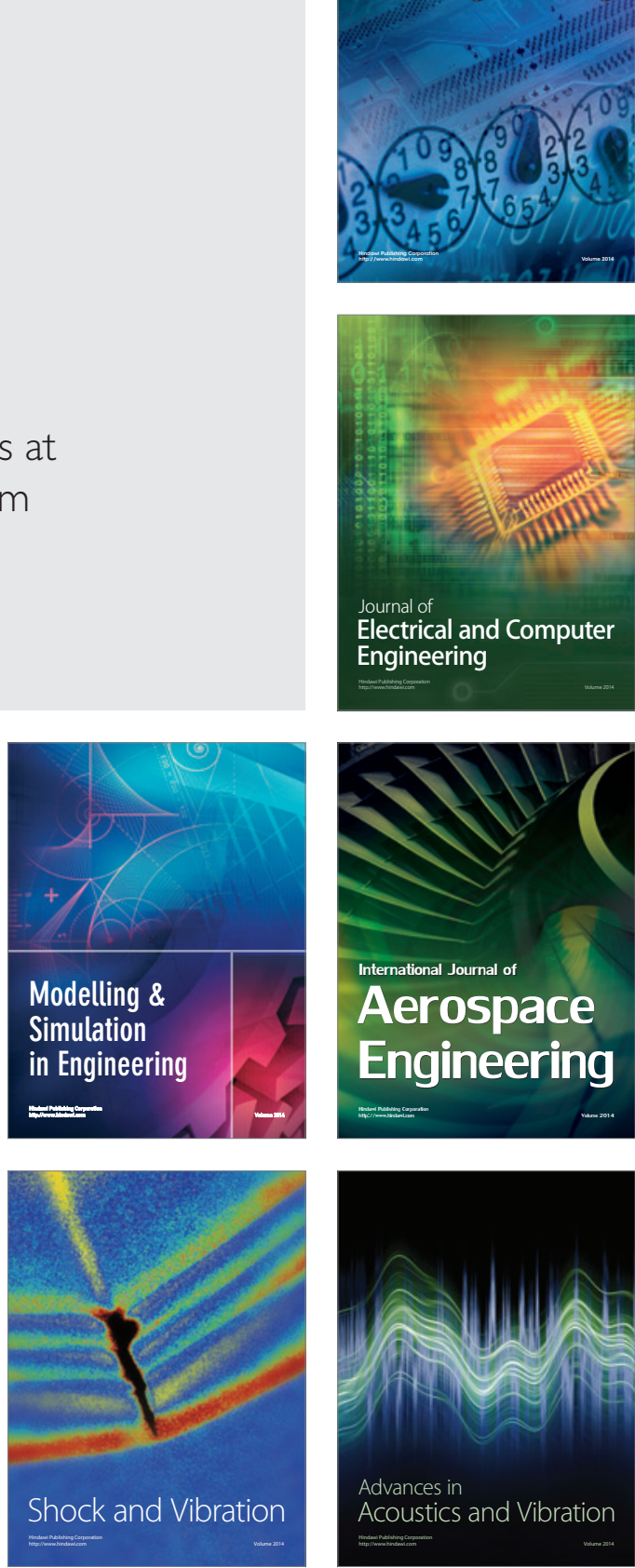